\title{
AVALIANDO O POTENCIAL DE APLICAÇÃO DO CONCEITO DE AGILIDADE NA GESTÃO DE TECNOLOGIA EM ICTS: O CASO EMBRAPII
}

Silvia Ronsom (sronsom@gmail.com) - Universidade de São Paulo, Departamento de Engenharia de Produção - EESC-USP - São Carlos-SP, Brasil.

Daniel Capaldo Amaral (amaral@ sc.usp.br) - Universidade de São Paulo, Departamento de Engenharia de Produção - EESC-USP - São Carlos-SP, Brasil.

\section{RESUMO}

O Gerenciamento Ágil de Projetos (GAP) é reconhecido nas áreas de desenvolvimento de produtos como uma abordagem gerencial apropriada para ambientes complexos, criativos e que necessitam de inovação. As Instituições Científicas, Tecnológicas e de Inovação (ICT) possuem estas características, mas ainda não há estudos sobre a agilidade nestas organizações. Seria possível avaliar a agilidade de ICTs? Investiga-se o tema por meio do estudo de caso da EMBRAPII, uma organização orquestradora de uma rede de ICTs que está inovando em métodos de gestão no Brasil. No artigo são apresentados revisão sobre o conceito de agilidade, um modelo conceitual e a sua aplicação para a avaliação da EMBRAPII. O resultado indica as características de agilidade em processos gerenciais de ICTs e da organização orquestradora e a possibilidade de se comparar a agilidade entre instituições de pesquisa.

Palavras-chave: GAP, ICT, agilidade, desempenho em agilidade, análise semântica 


\section{INTRODUÇÃ̃}

As organizações de Ciência e Tecnologia (C\&T) executam importante papel no cenário de inovação dos países. Elas sustentam a inovação comercial, fornecendo conhecimento científico (Manual de Oslo, 2005). Entre elas, estão as Instituições Científicas, Tecnológicas e de Inovação (ICT) ${ }^{1}$.

As ICTs são reconhecidas como ambientes de alta complexidade, por atuarem intermediando o desenvolvimento de novos conhecimentos e tecnologias para a aplicação no mercado. Rigby et al. (2016) identificaram características de complexidade em ambientes correlacionados com ICTs: soluções desconhecidas, especificações de projetos com mudança constante, avanços criativos e colaboração interfuncional. Entre várias abordagens gerenciais inerentes a esses ambientes, os autores defendem uma em especial - o Gerenciamento Ágil de Projetos (GAP).

Tal abordagem tem se destacado, especialmente em empresas de alta tecnologia e de desenvolvimento de software (Lee e Yong, 2010; Persson et al., 2012). Em relação à abordagem tradicional de gerenciamento de projetos, o GAP se diferencia pela busca da flexibilidade no planejamento e adequação às necessidades dos clientes, por meio de autogestão e simplicidade, sempre em busca de agregar maior valor ao projeto (Amaral et al., 2011). Quando uma equipe de projeto conquista um desempenho superior neste sentido, diz-se que a equipe ampliou o nível de agilidade (Conforto et al., 2016).

Um dos pesquisadores especialistas em estratégias de inovação, Prof. Marco Iansiti, afirma que esta capacidade ou habilidade é um dos diferenciais competitivos fundamentais para os atores de ecossistemas de inovação (Iansiti e Euchner, 2018, p. 12). Avaliar ou comparar as ICTs quanto a agilidade seria, assim, um importante instrumento para o avanço dos métodos gerenciais na área. Seria possível utilizar o construto "agilidade" no domínio das ICTs? Quais seriam suas principais características?

\footnotetext{
${ }^{1}$ Nomenclatura conforme o Art. $2^{\circ}$ da Lei $N^{\circ} 13.243$, de 11 de janeiro de 2016 (Brasil, 2016).
} 
Por meio de um estudo de caso da EMBRAPII - instituição de fomento à inovação que atua no Brasil, este artigo apresenta uma análise semântica inicial para potencial aplicação do conceito em ICTs.

\section{REVISÃO TEÓRICA}

Este trabalho busca estudar as ICTs sob a ótica de seus hábitos de ação, seus processos de negócio e rotinas de trabalho, que segundo Nelson e Nelson (2002) tratam-se da maneira de fazer algo, do curso das ações de instituições, em um sistema de inovação.

Para gerar novas tecnologias e produtos, as ICTs executam processos de negócio consagrados de Gestão de Tecnologia e Inovação, tais como: 1) Processo de Desenvolvimento de Tecnologia (PDT), ver, como exemplos: Sheasley (2000), Clark e Wheelwright (1993), Clausing (1993), Cooper (2006), Creveling et al. (2003), Schulz et al. (2000); 2) Processo de Desenvolvimento de Produto (PDP), ver: Pugh (1978), Clark e Wheelwright (1993), Clausing (1993), Ulrich e Eppinger (1995), Baxter (1998), Rozenfeld et al. (2006), entre outros; 3) outros mais generalistas relacionados à inovação, como maturidade e gerenciamento de portfólio e projetos: Davenport (1993), Hansen e Birkinshaw (2007), Salerno (2015), CMMI-DEV, PMBOK, Amaral et al. (2011), entre outros.

As teorias citadas foram desenvolvidas para projetos de produtos comerciais ou operações, mas as ICTs estão imersas em ambientes com características específicas. Por exemplo, Mohan e Rao (2005), discutem a crescente pressão social, a exigência sobre pesquisas úteis e o encolhimento dos orçamentos de pesquisa em Universidade. Eles defendem que os acordos entre partes interessadas, devem ser flexíveis suficientes para permitir uma revisão dos objetivos quando os parceiros são confrontados com evoluções inesperadas (Ingham e Mothe, 1998; Chiesa e Manzini, 1998).

Elas necessitam, portanto, gerenciar projetos com pouca previsibilidade, soluções desconhecidas em ambientes complexos, juntamente com características como necessidades de avanços criativos e colaboração interfuncional e, conforme Rigby et al. (2016), esse são aspectos de ambientes propícios para a abordagem GAP. Essas características são aderentes aos ambientes em que os métodos ágeis foram desenvolvidos.

Alguns estudos iniciais abordam gestão de tecnologia, por exemplo, Carlos (2015), identificou dificuldades de atualização no Processo de Planejamento Tecnológico e propôs um 
modelo utilizando conceitos de agilidade e inteligência competitiva, e, Conforto et al. (2016), apresenta um caso de aplicação de métodos híbridos de gerenciamento, ágil e tradicional, para o desenvolvimento de inovação. Nenhum dos autores discutiu, porém, o conceito de agilidade no contexto de ICTs, que é central na teoria de GAP.

\subsection{O conceito de agilidade e sua aplicação em ICTs}

O termo agilidade foi definido na área de gestão de projetos por Conforto et al. (2016, p.667) como uma habilidade de mudar produtos e plataformas tecnológicas rapidamente como respostas para partes interessadas.

Entretanto, existiriam estratégias diferentes entre os tipos de organismos envolvidos em ecossistemas de inovação, em que para alguns a agilidade seria fundamental para a sobrevivência (Iansiti e Euchner, 2018, p. 13). Isso reforça indícios de que existam lacunas na literatura quanto à investigação do "construto agilidade" para organizações de C\&T.

O termo "agilidade" foi observado pela primeira vez na área de manufatura (Nagel e Dove, 1991), onde foi disseminada como "manufatura ágil”. Em seguida, disseminou-se para a área de gerenciamento de projetos, com trabalhos como os de Boehm, 2002; Boehm; Turner, 2004; Decarlo, 2004; Schwaber, 2004; Augustine, 2005; Cohn, 2005; Hodgetts, 2005; Griffiths, 2005; manifesto ágil - Manifesto para Desenvolvimento Ágil de Software (BECK et al., 2001) e, para a melhoria dos métodos gerenciais na área de desenvolvimento de produtos (Chin, 2004; Highsmith, 2004; Smith, 2007; Cooper, 2008; Conforto; Amaral, 2009; 2010).

Conforto et al. (2016) realizaram um estudo amplo e, por meio do Quadro 2, apresentaram uma síntese de definições de agilidade, elaborado conforme uma técnica de análise denominada semântica de frames. 
Quadro 2 - Síntese de definições de agilidade de Conforto et al. (2016)

\begin{tabular}{|c|c|c|c|c|}
\hline $\begin{array}{l}\text { Sub-area } \\
\text { (Agilidade em) }\end{array}$ & Entidade & Evento & Grau & Motivador \\
\hline Organizações ${ }^{2 *}$ & Organização & \multirow{3}{*}{$\begin{array}{lr}\text { Habilidade } & \text { de } \\
\text { mudar } & \text { (ex. } \\
\text { plataformas } & \text { de } \\
\text { produtos } & \text { e } \\
\text { serviços) } & \end{array}$} & \multirow{4}{*}{ Rapidamente } & \multirow{2}{*}{$\begin{array}{l}\text { Respostas para } \\
\text { stakeholders ou } \\
\text { necessidades de negócio } \\
\text { tecnologia, competidores } \\
\text { novos mercados } \\
\text { demandas } \\
\text { oportunidades. }\end{array}$} \\
\hline Manufatura & Manufatura & & & \\
\hline $\begin{array}{l}\text { Desenvolvimento } \\
\text { de produtos }\end{array}$ & $\begin{array}{l}\text { Processo de } \\
\text { desenvolvimento de } \\
\text { produto }\end{array}$ & & & \multirow{2}{*}{$\begin{array}{l}\text { Resposta para clientes ou } \\
\text { para necessidades dos } \\
\text { stakeholders, demandas } \\
\text { de tecnologia ou mercado }\end{array}$} \\
\hline $\begin{array}{l}\text { Gerenciamento de } \\
\text { projetos }\end{array}$ & Time do Projeto & $\begin{array}{l}\text { Habilidade para } \\
\text { mudar o plano no } \\
\text { projeto }\end{array}$ & & \\
\hline
\end{tabular}

Os autores identificaram, a partir desta definição, possíveis variáveis para se medir a agilidade e, por meio de um estudo de campo em 180 empresas, identificaram dois fatores ou dimensões para este construto, segundo Conforto et al. (2016, p.670), são:

- Capacidade de mudar o plano do projeto (Flexibilidade). Depende de variáveis: tempo necessário para a tomada de decisão e esforço para alterar o plano e comunicálo. Está relacionado com a tecnologia que está sendo tratada, práticas gerenciais e organização do trabalho.

- Envolvimento ativo do cliente (Envolvimento). Está relacionado com a frequência com que o cliente entra em contato, o preparo para priorizar e ajudar a orientar as decisões e o seu nível de envolvimento no projeto.

Este desenvolvimento teórico do construto foi adotado como um ponto de partida para explorar o conceito de agilidade em ICT. 


\section{METODOLOGIA}

A metodologia de estudo de caso, segundo Gil (2008), estabelece os passos selecionados e explicados a seguir, já correlacionados com as atividades deste trabalho.

- Formulação do problema. O tema foi justificado por meio de revisão bibliográfica com as palavras-chave citadas no resumo. Conforme exposto na seção 2, foi possível identificar que não existem estudos prévios sobre o conceito de agilidade em ICTs.

- Definição da unidade-caso. A EMBRAPII e suas ICTs credenciadas foram definidas como unidade-caso devido as suas características de ambientes $C \& T$ e sua semelhança com as características de ambientes ágeis inicialmente destacados na teoria por Rigby et al. (2016).

- Elaboração do protocolo de análise. O protocolo foi elaborado a partir de um modelo conceitual, neste caso, o framework de análise semântica desenvolvido por Conforto et al. (2016), conforme apresentado na seção 2.1.

- Coleta de dados. Considerou estudos publicados sobre a EMBRAPII e material institucional disponível na internet. As fontes principais foram: Reynolds et al. (2019), Ronsom e Amaral (2017) e na EMBRAPII (2014).

- Método de análise dos dados. Foram categorizadas as informações segundo a definição de agilidade e as variáveis que medem a agilidade, segundo o modelo conceitual proposto por Conforto e Amaral (2016), apresentado na seção 2.

\subsection{Descrição do caso EMBRAPII}

A EMBRAPII vem se consolidando como uma agenciadora de inovação de alta eficiência no cenário brasileiro. Criada em 2013, ela credencia e gerencia uma rede de ICTs capazes de prospectar e desenvolver tecnologias inovadoras com empresas industriais. $\mathrm{O}$ seu modelo de operação é similar ao dos Institutos Fraunhofer na Alemanha, dos Institutos Carnot na França, e nos institutos Catapult na Inglaterra.

A EMBRAPII, conforme Reynolds et al. (2019, p. 231), possui características únicas entre agências de fomento nacionais:

- Planejamento e "agilidade": As ICTs credenciadas estabelecem um Plano de Ação (PA) melhorado ao longo do tempo com base no constante aprendizado com a indústria e no 
planejamento tecnológico. O PA tem foco de atuação, identificação de competências, potencial de mercado, possíveis parceiros e metas de desempenho.

- Crescimento e prospecção de mercado: As ICTs tem capacidade de atrair investimentos industriais, por meio de um processo de atualização e oferta de $\mathrm{P} \& \mathrm{D}$, focada na demanda industrial e na área de competência. O tempo médio de contratação de projetos é de um mês.

- Excelência em gestão: A EMBRAPII disponibiliza um guia de melhores práticas de gestão de P\&D para as ICTs ${ }^{3}$.

- Flexibilidade de orçamento: As ICTs podem planejar seus portfólios de projetos com aplicação de maior volume de recursos em projetos de maior risco.

- Flexibilidade em gestão de projetos: as ICTs têm autonomia para revisar constantemente os objetivos dos projetos e realizar mudanças alinhadas com as empresas.

- Contínua avaliação: A EMBRAPII mantém parceria com um grupo de acadêmicos e profissionais de indústrias para avaliar os projetos de $\mathrm{P} \& \mathrm{D}$ das ICTs.

Hoje a EMBRAPI conta com 42 ICTs credenciadas, 30 pessoas na administração em Brasília-DF e mais de 3.400 pesquisadores em projetos em todo o país. A seção a seguir mostra os elementos do conceito de agilidade nesta organização.

\section{RESULTADOS E DISCUSSÃO}

A análise do caso partiu do quadro semântico representado no Quadro 2, na seção 2.1 e foi complementada por características da EMBRAPII, citadas em Reynolds et al. (2019, p. 231) e informações disponíveis na internet. Considerando os conhecimentos apresentados de forma sucinta na seção 2, foi possível sugerir características genéricas de agilidade em ICTs. O resultado da análise está sintetizado no Quadro 3.

\footnotetext{
3 Ver Ronsom, S (2015); Ronsom e Amaral (2017); https://embrapii.org.br/institucional/manuais/sistema-de-excelenciaoperacional-embrapii/
} 


\section{de Desenvolvimento do Produto}

11 a 13 de setembro de 2019 - Universidade de Brasília UnB

Quadro 3 - Características de agilidade de ICTs credenciadas na EMBRAPII, segundo o framework de Conforto et al. (2016) (elaborado pela autora)

\begin{tabular}{|c|c|c|c|c|}
\hline \multicolumn{3}{|c|}{ Conforto et al. (2016) } & \multicolumn{2}{|c|}{ Elementos observados na descrição do funcionamento da EMBRAPII } \\
\hline Entidade & Evento & $\begin{array}{l}\text { Fatores de } \\
\text { agilidade }\end{array}$ & $\begin{array}{l}\text { Características identificadas no estudo, } \\
\text { com base em Reynolds et al. (2019) e informações disponíveis na internet }\end{array}$ & $\begin{array}{l}\text { Características genéricas sugeridas a } \\
\text { partir do estudo de caso }\end{array}$ \\
\hline & \multirow[b]{2}{*}{$\begin{array}{l}\text { Habilidade de } \\
\text { mudar (ex. } \\
\text { plataformas de } \\
\text { produtos e } \\
\text { serviços) }\end{array}$} & $\begin{array}{l}\text { Envolvimento } \\
\text { com o governo }^{4}\end{array}$ & $\begin{array}{l}\text { - acompanhamento pela Comissão de Acompanhamento e Avaliação (CAA) do } \\
\text { Contrato de Gestão, por meio de indicadores de desempenho, onde são discutidos } \\
\text { ajustes de rotas estratégicas para o país relacionadas a inovação. (Ver: } \\
\text { https://embrapii.org.br/wp-content/images/2019/02/Relato\%CC\%81rio-do-1\%C2\%BA-Semestre-de-2018- } \\
\text { CAA-aprovada-Ad-Rerendum-pelo-CA.pdf - p. 4,9 e 11) }\end{array}$ & $\begin{array}{l}\text { - Habilidade de contatar e obter } \\
\text { informações do Governo e } \\
\text { organizações governamentais. }\end{array}$ \\
\hline EMBRAPII & & $\begin{array}{l}\text { Flexibilidade } \\
\text { nos planos com } \\
\text { o governo }\end{array}$ & $\begin{array}{l}\text { - Entrada de novas áreas de competência para fomento no Contrato de Gestão, por } \\
\text { meio de parcerias com diversos Ministérios. } \\
\text { https://www.correiobraziliense.com.br/app/noticia/economia/2019/01/31/internas_economia,734330/embr } \\
\text { apii-que-ja-teve-r-1-bi-em-investimento-fomenta-inovacao.shtml } \\
\text { http://www.mctic.gov.br/mctic/opencms/salaImprensa/noticias/arquivos/2017/12/Parceria com o Ministe } \\
\text { rio da Saude abre leque de atuacao da Embrapii diz ministro.html } \\
\text { - novas parcerias de apoio à projetos de inovação para públicos específicos. } \\
\text { http://www.mctic.gov.br/mctic/opencms/salaImprensa/noticias/arquivos/migracao/2017/06/Acordo entre } \\
\text { Embrapii_e_Sebrae_estimula a inovacaonas_micro_e_pequenas empresas.html }\end{array}$ & $\begin{array}{l}\text { - Habilidade de ajustar estratégias em } \\
\text { nível de atuação nacional, com } \\
\text { fomento a novos públicos e novas áreas } \\
\text { de competência. }\end{array}$ \\
\hline $\begin{array}{l}\text { Organização } \\
\text { - ICT } \\
\text { credenciada } \\
\text { de apoio à } \\
\text { inovação }\end{array}$ & $\begin{array}{l}\text { Habilidade de } \\
\text { mudar (ex. } \\
\text { plataformas de } \\
\text { produtos e } \\
\text { serviços) }\end{array}$ & $\begin{array}{l}\text { Envolvimento } \\
\text { do cliente - } \\
\text { empresa } \\
\text { parceiro }\end{array}$ & $\begin{array}{l}\text { - “...as ICTs credenciadas são responsáveis por visitar potenciais clientes e ofertas } \\
\text { propostas de pesquisa para apoiar os processos de inovação das empresas” } \\
\text { Reynolds et al. (2019, p. 238) } \\
\text { - “...as ICTs têm autonomia para negociar ...com as empresas, contratos } \\
\text { cronograma financeiros. ” Reynolds et al. }(2019, \text { p. 237) } \\
\text { - “...as ICTs devem mostrar como planejam suas abordagens aos parceiros } \\
\text { industriais, por meio de visitas, reuniões e eventos” Reynolds et al. (2019, p. 243) } \\
\text { - “...as ICTs devem ter confiança de que suas propostas de pesquisa são relevantes }\end{array}$ & $\begin{array}{l}\text { - Habilidade de contatar e obter } \\
\text { informações de empresas usuárias da } \\
\text { tecnologia. }\end{array}$ \\
\hline
\end{tabular}

${ }^{4}$ Além de empresas, a EMBRAPII também tem interação com o Governo Federal. 


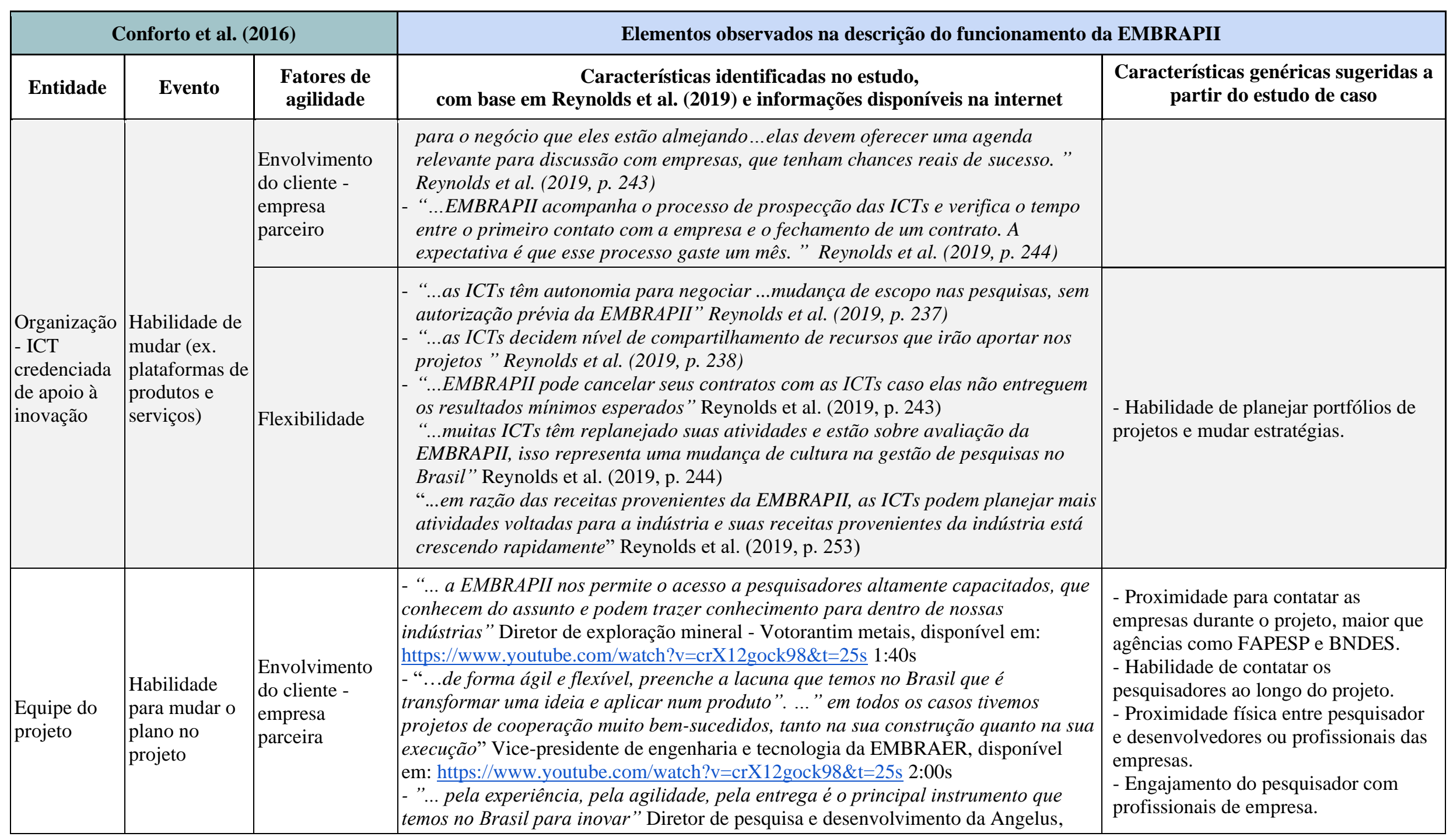




\begin{tabular}{|c|c|c|c|c|}
\hline \multicolumn{3}{|c|}{ Conforto et al. (2016) } & \multicolumn{2}{|c|}{ Elementos observados na descrição do funcionamento da EMBRAPII } \\
\hline Entidade & Evento & $\begin{array}{l}\text { Fatores de } \\
\text { agilidade }\end{array}$ & $\begin{array}{l}\text { Características identificadas no estudo, } \\
\text { com base em Reynolds et al. (2019) e informações disponíveis na internet }\end{array}$ & $\begin{array}{l}\text { Características genéricas sugeridas a } \\
\text { partir do estudo de caso }\end{array}$ \\
\hline & & & disponível em: https://www.youtube.com/watch?v=crX12gock98\&t=25s $5: 18 \mathrm{~s}$ & \\
\hline $\begin{array}{l}\text { Equipe do } \\
\text { projeto }\end{array}$ & $\begin{array}{l}\text { Habilidade } \\
\text { para mudar o } \\
\text { plano no } \\
\text { projeto }\end{array}$ & Flexibilidade & 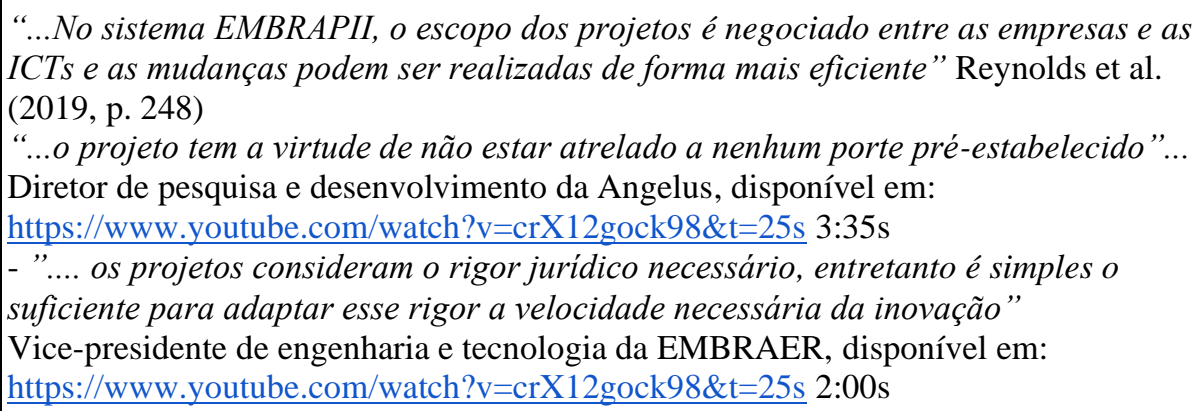 & $\begin{array}{l}\text { - Flexibilidade para alterar o objetivo } \\
\text { final do projeto. } \\
\text { - Flexibilidade para alterar as entregas } \\
\text { do projeto. } \\
\text { - Flexibilidade para alterar orçamentos } \\
\text { e previsões de gastos do projeto. }\end{array}$ \\
\hline
\end{tabular}




\section{Congresso Brasileiro de Inovação e Gestão}

\section{de Desenvolvimento do Produto}

11 a 13 de setembro de 2019 - Universidade de Brasília UnB

\section{CONCLUSÃO}

O artigo apresentou uma análise sobre o conceito de agilidade e sua aplicação em gestão de tecnologia para o caso EMBRAPII. O Quadro 3 mostra que foi possível caracterizar elementos que podem diferenciar esta agência de inovação de outras instituições similares.

Como consequência, este resultado indica que há uma possibilidade de que as ICTs, possam se diferenciar quanto a sua capacidade de responder rapidamente aos clientes. Em decorrência disso seria possível considerar que desempenhos distintos em agilidade poderiam impactar o desempenho de ecossistemas de inovação que possam ser explicados por este fator.

Portanto, o estudo indica a oportunidade para se investigar o nível de agilidade em ICTs e em agências de inovação. E que isso pode significar a possibilidade de desenvolver o conceito, por meio da qual, a agilidade para ICTs estaria relacionada a habilidade de mudar rapidamente e de forma inovadora as suas plataformas tecnológicas, produtos e serviços, com propósito de atingir melhor desempenho de seus portfólios de projetos e assim fornecer respostas tecnológicas diferenciadas para partes interessadas em ambientes complexos de inovação.

Estas são hipóteses que emergem deste caso e que demandam novos ciclos de pesquisa. Faz-se necessário considerar as características identificadas previamente neste estudo, revisões bibliográficas sistemáticas e métodos científicos de correlação de fatores, para revisar e confirmar a discussão sugerida por essa pesquisa inicial. Para o teste de campo, uma ferramenta de diagnóstico poderia ser desenvolvida. Outra sugestão seria a investigação de quais práticas, técnicas e ferramentas da abordagem ágil poderiam ser mais apropriadas para ICTs. Esses estudos podem contribuir tanto para a melhoria do desempenho dessas ICTs quanto para as teorias de gestão de tecnologia e redes de inovação. 


\section{REFERÊNCIAS}

Amaral, D. et al. Gerenciamento ágil de projetos: aplicação em produtos inovadores. São Paulo: Saraiva. 2011.

Augustine, S. Managing Agile Projects. Virginia: Prentice Hall PTR, 2005.

Baxter, M. Projeto de produto: guia prático para o desenvolvimento de novos produtos. São Paulo: Editora Edgard Blücher Ltda., 1998.

Beck, K. et al. Manifesto for agile software development. 2001. Disponível em < https://www.manifestoagil.com.br/ >. Acesso: em 16 de janeiro de 2019.

Boehm, B. Get ready for agile methods, with care. IEEE Computer Magazine, January, p.6469, 2002.

Boehm, B.; Turner, R. Balancing Agility and Discipline: a guide for the perplexed. AddisonWesley: Boston, 2004.

Brasil. Lei $\mathrm{N}^{\circ}$ 13.243, de 11 de janeiro de 2016. Dispõe sobre estímulos ao desenvolvimento científico, à pesquisa, à capacitação científica e tecnológica e à inovação. <http://www.planalto.gov.br/ccivil_03/_ato2015-2018/2016/lei/113243.htm>. Acesso em $10 \mathrm{de}$ julho de 2018.

Carlos R. Modelo para atualização de roadmaps utilizando conceitos de agilidade e inteligência competitiva. Dissertação (mestrado). Programa de Pós-graduação de Engenharia de Produção. Escola de Engenharia de São Carlos da Universidade de São Paulo. 2015.

Chiesa, V., Manzini, R., 1998. Organizing for technological collaborations: a managerial perspective. R\&D Manage. 28 (3), 199. 1998.

Chin, G. Agile Project Management: how to succeed in the face of changing project requirements. Amacom: New York, 2004

Clark, K. B.; Wheelwright, S. C. Managing new product and process development: text and cases. New York: The Free Press, 1993.

Clausing, D. Total quality development: a step-by-step guide to world-class concurrent engineering. American Society of Mechanical Engineers, New York, 1993.

CMMI. Improving process for better products. CMMI-DEV, V1.2, Software Engineering Institute, Pittsburg, 2006.

Cohn, M. Agile Estimating and Planning. Prentice Hall PTR: New York, 2005.

Conforto, E. C.; Amaral, D. C. Applying agile project management approach to NDP: the case of a small technology-based enterprise. In Proceedings of the 16th International Product Development Management Conference, Enschede. 2009. 
Conforto, E. C.; Amaral, D. C. Evaluating an agile method for planning and controlling innovative projects. Project Management Journal, 41(2), 73-80. 2010a.

Conforto E. C.; Amaral D. C. Agile project management and stage-gate model - A hybrid framework for technology-based companies. Journal of Engineering and Technology Management. 40. p 1-14. 2016.

Conforto et al. The agility construct on Project management theory. International Journal of Project Management. 34, 660-674. 2016.

Cooper, R. G. Managing technology development projects. Research Technology Management, 49, 6, 2006.

Cooper, R. Perspective: the stage-gate idea-to-launch process - update, what's new, and NexGen Systems. Journal of Product Innovation Management, 25, 3, 213-232, 2008.

Creveling, C. et al. Design for six sigma: in technology \& product development. New Jersey: Prentice Hall PH, 2003.

Davenport, T. Process Innovation: Reengineering Work Through Information Technology, 1993.

DeCarlo, D. Extreme Project Management: using leadership, principles, and tools to deliver value in the face of volatility. Jossey Bass: San Francisco, 2004.

EMBRAPII. Sistema de Excelência Operacional EMBRAPII. 2014. < https://embrapii.org.br/institucional/manuais/sistema-de-excelencia-operacional-embrapii/>. Acesso em: 30 de abril de 2019.

EMBRAPII. Empresários elogiam o modelo EMBRAPII de negócios. 2017. (5m53s). Disponível em: 〈https://www.youtube.com/watch? $\mathrm{v}=\mathrm{crX} 12$ gock98\&t=25s $>$. Acesso em: $22 \mathrm{de}$ maio de 2019.

Gil, A. Como elaborar projetos de pesquisa. Atlas: São Paulo, 2007.

Griffiths, M. Teaching agile project management to the PMI. IEEE Computer Society. In: Proceedings of Agile Development Conference, 2005.

Hansen, T.; Birkinshaw, J. The innovation value chain. Harvard Business Review. 2007.

Highsmith, J. Agile Project Management: creating innovative products. Addison-Wesley: Boston, 2004.

Hodgetts, P. Refactoring the development process: experiences with the incremental adoption of agile practices. IEEE Computer Society. In: Proceedings... Agile Development Conference, 2005 .

Iansiti, M.; Euchner, J. Competing in ecosystems. Research-Technology Management. 61(2), 10-16, 2018. 
Ingham, M.; Mothe, C. How to learn in R\&D partnerships. R\&D Management. 28 (4), 249. 1998.

Lee, S., Yong, H.S. Distributed agile: project management in a global environment. Empirical Software. Eng. 15, 204-217. 2010.

Mohan, S.R.; Rao, A.R. Strategy for technology development in public R\&D institutes by partnering with the industry. Technovation. 25 (12), 1484-1491. 2005.

Nagel, R.; Dove, R. 21st Century Manufacturing. Enterprise Strategy. Lehigh University, Iacocca Institute. 1991.

Nelson R.; Nelson K. Technology, institutions, and innovation systems. Research Policy. Vol 31. P 265-272. 2002.

ORGANIZAÇÃO PARA A COOPERAÇÃO E DESENVOLVIMENTO ECONÔMICO OCDE. Manual de Oslo: proposta de diretrizes para coleta e interpretação de dados sobre inovação tecnológica. 3a ed. Brasília: OCDE: FINEP, 2005.

Persson, J.S.; Mathiassen, L.; Aaen, I., 2012. Agile distributed software development: enacting control through media and context. Inf. Syst. J. 22, 411-433.

PROJECT MANAGEMENT INSTITUTE - PMI. A guide to the project management body of knowledge. (PMBOK). 4o edition. Pennsylvania: Project Management Institute.2008.

Pugh, S. Total design: integrated methods for successful product engineering. Reading, HA: Addison, 1978.

Reynolds E. B.; Schneider B. R.; Zylberberg E. Innovation in Brazil, Advancing Development in the 21st Century. Routledge. 2019.

Rigby D; Sutherland J.; Takeuchi H. Embracing Agile. Harvard Business Review. 2016

Ronsom, S. Proposta de padrão para sistema de gestão da inovação: a experiência EMBRAPII para a melhoria de um Sistema Nacional de Inovação. 2015. 134f. Dissertação (Mestrado em Engenharia de Produção) - Escola de Engenharia de São Carlos, Universidade de São Paulo, São Carlos, 2015.

Ronsom, S.; Amaral, D. C. Avaliação de redes de instituições de pesquisa científica e tecnológica baseada em um sistema de gestão padronizado. Gestão e Produção. 24(3). São Carlos, 2017.

Rozenfeld, H. et al. Gestão de desenvolvimento de produtos: uma referência para melhoria do processo. São Paulo: Saraiva, 2006.

Salerno, M. et al. Innovation processes: Which process for which project? Technovation, 35, 1, 59-70, 2015.

Schulz, A. et al. Development and integration of winning technologies as key to competitive advantage. Systems Engineering, 03, 04, 180-211, 2000. 
Schwaber, K. Agile Project Management with SCRUM. Microsoft Press: Washington, 2004.

Sheasley, W. D. Taking an options approach to new technology development. Research Technology Management, 43, 6, 37-43, 2000.

Smith, P. G. Flexible Product Development - building agility for changing markets. JosseyBass: San Francisco, 2007.

Ulrich, K.; Eppinger, S. Product design and development. New York, McGraw Hill, 1995. 